\title{
ISLAM E OCCIDENTE NEL NOME DELL'UMANESIMO
}

\author{
Carmela Baffioni
}

\begin{abstract}
RESUMO - O presente artigo compara algumas características bem conhecidas do humanismo ocidental com aquelas do assim chamado "humanismo muçulmano" dos séculos X-XII. A "idade de ouro" muçulmana, em suas várias facetas (filosofia, ciência, literatura, política, etc.), construiu-se sobre uma consistente, apesar de multifacetada, base religiosa. Razões históricas e culturais demonstram sempre que a ética não é suficiente a fim de estabelecer uma base comum para o diálogo com o Islã, e que é preciso aproximar-se dele principalmente pelo viés do pensamento religioso. O re-pensar do Livro Sagrado sobre um novo fundamento, proposto por diversos membros da intelligentzia muçulmana, e que realça, assim, o antigo humanismo, deve, pois, ser levado em consideração pela contraparte ocidental. Já o Ocidente, pelo contrário, não pode cometer o erro de ler o texto do Islã de acordo

ABSTRACT - The article compares some wellknown features of Western humanism with those of the so-called Muslim humanism (X-XII centuries). The Muslim "golden age" in its various aspects (philosophy, science, literature, politics, etc.) is built on a consistent, though multifarious, religious basis. Even cultural and historical reasons demonstrate, then, that ethics is not sufficient for establishing a common ground for dialogue with Islam, and that Islam has to be approached mainly in its religious meaning. A re-thinking of the Sacred Book on a new basis, proposed by many of contemporary Muslim intellighenzia, who also often emphasize the importance of ancient humanism, should be taken into account also by their Western counterpart. West should not, on the contrary, make the mistake of reading Islam according to its own historical and/or ideological attitudes.
\end{abstract} com suas (ocidentais) próprias atitudes históricas e/ou ideológicas.

PALAVRAS-CHAVE - Humanismo islâmico. Humanismo ocidental. Diálogo de culturas. Religião.

KEY WORDS - Muslim humanism. West humanism. Intercultural dialog. Religion.

L'ideale dell'umanesimo, basato sulla centralità dell'uomo e della sua libertà, viene oggi considerato una fra le vie possibili per sfuggire alla profonda crisi morale, di identità, economica e sociale - in cui versa l'Europa, in quanto portato-

Professore ordinario di Storia della filosofia islamica, Università degli Studi di Napoli "L'Orientale", Dipartimento di Studi e Ricerche su Africa e Paesi Arabi, Palazzo Corigliano, Piazza San Domenico Maggiore 12, 80134 Napoli. Questo studio rielabora parte della relazione "L'humanisme islamique dans l'âge d'or", da me tenuta alla Conférence Thématique su "Universalité de l'Humanisme" (Séminaire de contact Comenius, L'action de réseau pour l'Europe de la connaissance), svoltasi a Napoli, presso l'Istituto Italiano per gli Studi Filosofici, dal 14 al 18 novembre 2006.

\begin{tabular}{|l|l|l|l|l|l|}
\hline VERITAS & Porto Alegre & v. 52 & n. 3 & Setembro 2007 & p. 159-169 \\
\hline
\end{tabular}


re di una nuova forma di solidarietà fra gli uomini. A fronte di dolorosi squilibri, di confusioni e violenze a tutti i livelli, a fronte persino del paventato rischio di una catastrofe globale - nucleare o causata da irreparabili squilibri ecologici -, ai "nuovi umanisti" appare infatti soprattutto preoccupante lo scontro in atto fra le diverse culture, ingigantito negli ultimi anni da celebri intellettuali e dai media, dopo il forse troppo esaltato libro di Samuel Huntington, del 1996. ${ }^{1}$ Non essendo ammissibile infatti, per il "nuovo umanesimo", alcuna gerarchia fra le diverse civiltà, si può legittimamente aspirare ad un'osmosi fra culture, in quanto da ogni cultura, e non solo da quelle occidentali, è asserita la centralità dell'essere umano con la sua dignità. Dunque, anche se a tale enunciazione si giunge per vie diverse dalle nostre, se ne potranno individuare modelli, personali e sociali, secondo i quali realizzare quelle istanze, oggi calpestate, di libertà, di fratellanza, di pace, e ne scaturiranno, si pensa, il riconoscimento dell'uguaglianza di tutti, la valorizzazione delle diversità personali e culturali, l'esigenza di sviluppare le conoscenze al di là di quanto sinora accettato come verità assoluta, la libertà di professare qualunque idea o credenza, il totale ripudio della violenza. In ultima istanza il convincimento umanista, secondo cui ogni cultura e civiltà, ciascuna con la sua propria identità, può contribuire alla formazione di una nuova "nazione umana universale", ambisce a confutare la recente convinzione che, in un mondo in cui tutte le culture interagiscono, diventa difficile riconoscere cosa è bene e cosa è male e tutto - si dice con un'espressione di moda - si "relativizza".

Sempre sulla base dell'idea che non possono esserci fratture nello sviluppo di una civiltà, nulla osta a che anche l'Islam sia accolto all'interno del progetto dei "nuovi umanisti". D'altro canto nessuno oggi, neo-umanista o non, può negare l'assoluta necessità dell'Occidente di confrontarsi col mondo islamico, con un'urgenza sconosciuta fino a pochi anni fa. Vale dunque la pena di indagare se e in quale misura l'Islam condivida, o abbia condiviso, gli ideali dell' "umanesimo".

Di solito, il terreno comune fra Occidente e Islam sembra essere individuato nell'etica, e di fatto il Corano è ricco di suggestioni che illustrano il rapporto dell'uomo non solo col Creatore, ma anche con i suoi simili e persino con l'ambiente e le creature non umane. Perciò, non c'è dubbio che sia ben possibile evidenziare idee condivisibili sulle quali lavorare, come il rifiuto dello sfruttamento dell'uomo sull'uomo, dell'oppressione economica o politica, dell'emarginazione del diverso. È dunque su tali basi che si immagina che Islam e Occidente possano entrambi affrontare le sfide della modernità, non per farsene assorbire, ma per discernerne e potenziarne gli elementi positivi.

Questo, almeno, il punto di vista dell'Occidente laico, che oggi va ricordando costantemente i caratteri basilari dell'umanesimo del '400, quali la collocazione

Cf. S.P. Huntington, The Clash of Civilization and the Remaking of World Order, New York, Simon and Schuster. 
dell'uomo al centro della realtà in aperta opposizione al modello teologico medievale o il ritorno alla civiltà classica come alla più alta, attraverso uno studio approfondito del latino e il grande impulso alla ricerca filologica e storica. Ma, soprattutto, viene enfatizzato lo svincolamento dello studioso dall'autorità tradizionale, nel nome del senso storico e dell'autonomia critica che gli sarebbero assicurati dalla sua alta cultura: nell'umanesimo, lo studio dei classici prescinde totalmente da quella riduzione a schemi morali di cui, in ossequio alle esigenze della religione, era stato fatto oggetto nel Medioevo.

In verità, sotto l'urgenza della lotta contro le eresie, nel Medioevo erano state tentate conciliazioni della filosofia (sia pure come ancilla theologiae) con i testi sacri, per cercare giustificazioni razionali ai dogmi religiosi. Ma si trattava soprattutto di filosofia aristotelica, dalla quale era scaturita la sussunzione dell'idea di un universo finito, e della teoria del geocentrismo. L'umanesimo ripropone invece il platonismo, che meglio dell'aristotelismo sembra favorire l'idea della centralità dell'uomo nella riflessione, così come nella realtà; afferma, contro l'ipotesi tolemaica, un universo infinito privo di centro; e sviluppa il principio della libertà, sia morale sia intellettuale. Di conseguenza, viene spezzata l'unità enciclopedica medievale, per la quale tutte le scienze non erano che gradi successivi necessari per attingere, alla fine, la verità religiosa; le singole discipline cominciano a godere di autonomia, appunto per consentire all'uomo di conoscere e dominare le leggi della natura e della storia. ${ }^{2}$ Tutto ciò determina anche un enorme sviluppo delle scienze esatte e applicate, che va ad aggiungersi alla fioritura delle grandi biblioteche, come pure delle nuove figure professionali variamente connesse alla produzione e alla diffusione del libro.

Com'è ben noto, tutti questi elementi indussero a parlare di "Rinascimento", perché si ritenne di essersi liberati dalle diverse forme della "barbarie". L'interesse per l'individuo nella sua totalità implicò, d'altra parte, che non venisse neppure tralasciato il problema religioso. Mentre, con Machiavelli, la morale si svincolava dalla politica, la polemica contro la corruzione del clero e la formulazione di una nuova religiosità fondata sull'esperienza interiore ${ }^{3}$ portavano alla Riforma protestante.

\footnotetext{
Tuttavia - è stato pure notato - l'ideale umanista non fu esente da contraddizioni: mentre si affermava la dignità e l'autonomia dell'uomo, gli studiosi diventavano dei cortigiani al servizio delle signorie, che consideravano la cultura per lo più come un'elegante forma di pubblicità, o come un mezzo di evasione; il fondamentale individualismo si scontrava con i propugnati ideali di rinnovamento socio-culturale; nonostante l'esistenza di condizioni che andavano favorendo la nascita di una nuova economia capitalistica, si ravvisavano nel contempo le prime gravi avvisaglie di decadenza; e al grande progresso economico corrispondeva lo sfruttamento di contadini e artigiani. D'altra parte, l'umanesimo non avrebbe potuto non riconoscere la funzione dei monasteri dell' "età di mezzo" quali scrigni delle vestigia della cultura classica.

L'ideale della renovatio, che implicava la lettura diretta dei testi sacri e l'indifferenza verso il formalismo del culto.
} 
Quali di questi elementi possono, in un modo o nell'altro, essere riconosciuti nell'Islam? Questo studio verterà sull'Islam classico, quello del cosiddetto "periodo aureo", anzitutto per la sua ricchezza culturale, che può quindi più facilmente giustificare la nostra indagine, ma anche perché è in gran parte con l'Islam classico che si misura pure l'intellighenzia islamica di oggi, tanto nella riflessione sul proprio passato quanto nelle sue varie posizioni nei confronti dell'Occidente; e, infine, perché sono quelli dell'Islam classico gli aspetti maggiormente enfatizzati dai nuovi umanisti.

Tuttavia, non foss'altro che perché tale periodo si estende dal X al XII/XIII secolo, e corrisponde perciò in pieno al nostro Medioevo, le caratteristiche fondamentali dell'umanesimo originario non possono essere del tutto adattate alla civiltà islamica, anzitutto per quanto concerne la posizione di radicale alterità che esso assunse nei confronti di quella che volle chiamare l'"età di mezzo". Ma che dire allora della "knowledge triumphant" di quest'epoca, che - a illustrazione del prestigio in cui l'Islam tenne le conoscenze filosofiche, scientifiche, letterarie, artistiche - ha dato il titolo a un celeberrimo libro di Franz Rosenthal? ${ }^{4}$

In questi secoli si dispiega, in effetti, la parte più feconda della civiltà islamica, quella a proposito della quale alcuni studiosi - il più noto dei quali è Joel Kraemer $^{5}$ - hanno ritenuto di poter legittimamente spendere le categorie di "umanesimo" e di "rinascimento". Varrà dunque la pena di segnalarne alcuni elementi caratterizzanti, per porre questi secoli a confronto con quelli che furono caratterizzati dall'umanesimo occidentale.

Il grande sviluppo intellettuale dei "secoli d'oro" dell'Islam fu anch'esso favorito dalle fiorenti condizioni economiche, assicurate dai commerci e dalla tassazione. Il grado di urbanizzazione era altíssimo, ${ }^{6}$ né ci fu in Islam opposizione fra la cultura urbana ed altre culture come, ad esempio, quella che si determinò in Europa fra gli abitanti dei monasteri e quelli dei castelli feudali; gli stessi teologi vivevano nelle città.

Gli Abbasidi, in opposizione agli Umayyadi, avevano promosso un universalismo basato non più sull'appartenenza tribale, ma su quella religiosa. Ciò contribuì all'integrazione, nella comunità musulmana, di vaste popolazioni dalle origini più diverse, tra le quali particolare rilevanza per lo sviluppo della filosofia e delle scienze ebbero quelle iraniche e centroasiatiche. A tale integrazione fa ris-

\footnotetext{
Cf. F. Rosenthal, Knowledge Triumphant. The Concept of Knowledge in Medieval Islam, Leiden, Brill, 1970.

5 Cf. J.L. Kraemer, Philosophy in the Renaissance of Islam: Abū Sulaymān al-Sijistānī and his Circle, Leiden, Brill, 1986; id., Humanism in the Renaissance of Islam. The Cultural Revival during the Buyid Age, Second Revised Edition, Leiden - New York - Köln, Brill, 1992.

6 Nelle tre maggiori città della Mesopotamia e nelle due più grandi dell'Egitto abitava quasi il 20\% di tutta la popolazione. Per percentuale di abitanti di città con più di centomila abitanti, la Mesopotamia e l'Egitto dei secoli VIII e X superarono paesi dell'Europa occidentale del secolo XIX quali i Paesi Bassi, l'Inghilterra o la Francia. Baghdad poteva avere quattrocentomila abitanti e Fustat, Cordova, Alessandria, Kufa e Bassora fra i cento e i duecentomila.
} 
contro la promozione di incontri-dibattito fra dotti di diversa provenienza e ideologia, i cosiddetti majālis, che si tenevano al cospetto del califfo.' Anche la cultura islamica fu strettamente legata alla vita di corte: letterati, filosofi e scienziati sono molto spesso appoggiati dai vari regimi, magari con alterne fortune.

Naturalmente, per l'intellighenzia tale universalismo non mancò di assumere una connotazione razionalistica - una sola è la verità che si rivela alla ragione umana -, al punto che alcuni pensatori approdarono addirittura allo scetticismo religioso. ${ }^{8}$ Il termine adab, che ora caratterizza la classe di dotti, indicava in origine le norme di comportamento dei beduini, ma assume un significato in senso "umanista" allorché il califfato diventa il centro di interrelazione fra diverse tradizioni, culturali e confessionali. Esso allude dunque alla raffinatezza e all'esprit della vita cittadina.

Tutta questa fioritura culturale era stata, naturalmente, preparata dal periodo precedente, che aveva visto il recupero della filosofia e delle scienze straniere, soprattutto greche. Tale periodo culminò nel IX secolo, sotto il califfato abbaside, ma era già stato avviato nell'VIII, sotto gli Umayyadi - benché le testimonianze al riguardo siano tutt'altro che numerose. Gli studiosi preposti a tali attività ebbero modo di dimostrare una grande acribia filologica - ampiamente documentata dai bio-bibliografi - tanto nella fase delle traduzioni come pure nel corso della ricerca e della collazione dei manoscritti. Anche in Islam nacquero biblioteche e centri di studio, e si formò una nutrita classe di scribi e di addetti alla produzione del libro.

Ed è in questi decenni che si sviluppa anche la Mu'tazila, ovvero la corrente teologica più razionalista dell'Islam. Vale la pena di segnalare, in questo contesto, la sua teorizzazione del libero arbitrio, fondata sul principio della "giustizia" divina: contro l'ortodossia ash'arita, la quale attribuisce a Dio una libertà e un volontarismo che sfociano nell'arbitrio più assoluto, si ritiene cioè che Dio fa qualcosa perché è giusta, e non che qualcosa è giusta perché Dio la fa.

\footnotetext{
Cfr. H. Lazarus-Yafeh, M.R. Cohen, S. Somekh, S.H. Griffith eds., The Majlis. Interreligious Encounters in Medieval Islam, Wiesbaden, Harrassowitz, 1999.

8 Cfr. D. Urvoy, Les penseurs libres dans l'Islam classique, Paris, Michel, 1996 e S. Stroumsa, Freethinkers of Medieval Islam, Leiden, Brill, 1999. Mi piace tuttavia ricordare l'interesse che già negli anni trenta del secolo scorso il nostro Francesco Gabrieli dimostrò per autori quali Abū 'l-'Alā' al-Ma'arrī o Ibn al-Muqaffa', che si potrebbero in qualche modo considerare "liberi pensatori". Cfr. ad esempio L'opera di Ibn al-Muqaffa", in "Rivista degli Studi Orientali", 13, 1931-32, pp. 197-247; La "Zandaqa" au I siècle abbasside, in L'élaboration de l'Islam, Colloque de Strasbourg 12-13-14 juin 1959 (Travaux du Centre d'Études Supérieures spécialisé d'Histoire des Religions de Strasbourg), Paris, Presses Universitaires de France, 1961, pp. 23-38 ; Imâmisme et littérature sous les Bûyides, in Le Shî'isme imâmite, Colloque de Strasbourg 6-9 mai 1968 (Travaux du Centre d'Études Supérieures spécialisé d'Histoire des Religions de Strasbourg), Paris, Presses Universitaires de France, 1970, pp. 105-113. Tuttavia, ad onta della sua ben nota ammirazione per la civiltà persiana, lo studioso non sembra aver avuto alcuna predilezione per la letteratura araba "eretizzante" di origine sciita (cfr. ad esempio La tradizione iranica nella civiltà musulmana, in "La parola del passato", 27, 1972, pp. 89-99).
} 
Bisogna tuttavia anche ricordare che il recupero dei classici avvenne inizialmente per preoccupazioni o religiose (apologetiche, sia interne che esterne) o politiche; per questo secondo aspetto soprattutto con gli Abbasidi, che attraverso il collegamento alla Grecia classica speravano di marcare ulteriormente la loro opposizione agli Umayyadi, fortemente legati invece, e influenzati, dalla Grecia bizantina e cristiana. ${ }^{9}$ Quanto ai contenuti di tale recupero - del quale non va dimenticato che fu, per secoli, la nostra sola fonte di conoscenza dei classici antichi -, essi hanno in genere una corrispondenza quasi assoluta agli "originali" dei quali disponiamo oggi. I pochi "adattamenti" si spiegano soprattutto con la Weltanschauung religiosa dei nuovi fruitori, che portò alla strategica sostituzione di alcuni termini chiave: più che di "causa" o di "principio primo" si parlò, ad esempio, di "bene puro"; l'eimarmène fu letta in termini di pronoia, e così via. ${ }^{10}$ Per quanto riguarda la $M u^{\prime}$ tazila, d'altra parte, nella sua teorizzazione del libero arbitrio è del tutto assente l'idea della libertà morale: l'uomo deve adeguare il suo comportamento ai dettami della Legge divina.

Può essere anche interessante accennare alla politica. Grandissima parte del pensiero politico dell'Islam classico si fondò su un principio attribuito ad Ardashīr ibn Bābak, il primo re sasanide (III sec. a.C.). Nella sua forma originaria, esso recitava: "giustizia e regno sono fratelli gemelli", ma l'adagio islamico recita che "religione e regno sono fratelli gemelli". È evidente in questa sostituzione il peso dell'idea islamica in cui potere religioso e potere politico sono, fin dalle origini, riuniti in una sola persona, il Profeta, e dunque, di fatto, l'inscindibilità della politica dalla religione. ${ }^{11}$

E per tornare ai "secoli d'oro", la tanto decantata verità razionale era destinata ad un'assoluta minoranza di "eletti", cui veniva contrapposta la "massa", per la quale la verità era attingibile solo tramite la Rivelazione. E mentre a una conciliazione fra fede e ragione lavorò costantemente la filosofia islamica, non va dimenticato che l'ortodossia, sempre in nome dell'assoluta libertà e volontarismo divini, rifiutò persino il concetto di filosofia come scienza delle cause, e dunque l'aristotelismo (anche se ciò non impedì il progresso degli studi scientifici e tecni-

9 Cfr., per questa tesi, soprattutto D. Gutas, Greek Thought, Arabic Culture. The Graeco-Arabic Translation Movement in Baghdad and Early 'Abbäsid Society (2th-4th /8th -10th centuries), London-New York, Routledge, 1998.

10 Ben lungi dal configurarli come fraintendimenti o, peggio, come falsificazioni, tali adattamenti risentono comunque in primo luogo delle interpretazioni dei commentatori tardo-antichi, talora cristiani, e di quelle dei primi traduttori, che volgevano i testi greci in siriaco, e poi dal siriaco all'arabo, ed erano nestoriani o monofisiti. Mi permetto di rinviare, al riguardo, al mio recente studio Trasmissione e ricezione dei testi filosofici nel mondo arabo e latino, in M. Capaldo, F. Cardini, G. Cavallo, B. Scarcia Amoretti (edd.), Lo spazio letterario nel Medioevo, 3. Le culture circostanti, vol. II, La cultura arabo-islamica, Roma, Salerno Editrice, 2003, pp. 499-529.

${ }^{11}$ Cfr. F. Rosenthal, Political Justice and the Just Ruler, in J.L. Kraemer and I. Alon eds., Religion and Government in the World of Islam. Proceedings of the Colloquium held at Tel-Aviv University 3-5 June 1979 (Israel Oriental Studies X), Tel-Aviv, Tel-Aviv University, 1983, pp. 92-101. 
ci, sia per esigenze pratiche o connesse proprio all'esecuzione di determinati doveri religiosi, ${ }^{12}$ sia perché tali studi erano considerati, dal punto di vista religioso, meno "pericolosi" che non quelli di metafisica).

I circoli di filosofia e scienze fiorirono dunque soprattutto attorno alle corti del $\mathrm{X}$ e dell'XI secolo, che erano corti sciite. A fronte dell'assenza di culto e di magistero ufficiale nell'Islam ortodosso (ad eccezione di quello dei 'ulema), gli Sciiti una docenza ce l'hanno, e per di più estremamente vincolante: si tratta della docenza dell'imām, del "califfo" (= vicario) del Profeta, che per loro dev'essere un discendente diretto da Muhammad, in quanto destinato ad ereditarne la conoscenza esoterica del Libro sacro. ${ }^{13}$ L'esplicitazione dei significati esoterici apre la via a un'indagine razionale del Corano, e spesso le interpretazioni razionali vengono dagli Sciiti radicalizzate in direzione allegorico-simbolica. Ciò offrì la più gran parte della materia propria delle filosofie sciite.

Naturalmente, se il punto di partenza continua ad essere il Libro sacro, anche per le élites filosofeggianti il maestro supremo continua ad essere Dio, e ancora Dio è l'obiettivo finale della ricerca filosófica. ${ }^{14}$ Per gli Ismailiti, addirittura - i più radicali degli Sciiti -, nonostante le loro ricche elaborazioni teoretiche, la salvezza (che già per i filosofi coincide con la conoscenza di Dio) non si ottiene se non con l'aiuto dei Profeti, degli imām e di "coloro che ne prendono il posto". E resta pure da segnalare l'enfasi data dall'ismailismo alle diverse figure professionali, fino a costituirle in vere e proprie gilde. ${ }^{15} \mathrm{Ma}$ si tratta di un aspetto solo in apparenza dissonante: in realtà, determinate competenze tecniche - quelle, ad esempio, dei fabbri, degli orefici, dei tintori, dei lavoratori del vetro - erano la base imprescindibile per lo sviluppo di scienze esoteriche, prima fra tutte l'alchimia.

${ }^{12}$ Si pensi anche soltanto all'esigenza di volgersi alla Mecca durante la preghiera, allo stabilimento delle fasi lunari e al rilevamento della luna nuova per dare inizio al digiuno del Ramaḍān, agli spostamenti per adempiere al pellegrinaggio.

13 Tale significato esoterico si ritiene comunicato da Dio anche al Profeta - il che ne garantisce la superiorità rispetto all'imām -, il quale però non ebbe il mandato di rivelarlo agli uomini.

14 Viene talora anche sviluppata un'ideologia dell'amore (cara, pur nei termini loro propri, anche ad alcuni celebri esponenti del Rinascimento, come Marsilio Ficino). Al-Āmir̄̄, ad esempio, propugna l'ideale dell'amore, con riferimento al rapporto del governante con i sudditi, del padre con i figli, del marito con la moglie, e, infine, dell'essere umano con i suoi concittadini, con l'umanità e con gli esseri viventi in genere; al-Tawḥ̄ì̄ osserva da parte sua che il dualista o il materialista sentono il bisogno di agire bene, anche senza aspettarsi quel tipo di ricompensa suggerito dalla rivelazione religiosa. Su Abū 'l-Ḥasan al-Āmir̄̄ e Abū Hayyān al-Tawḥ̄ì̄ī cfr. ora C. D'Ancona (ed.), Storia della filosofia nell'Islam medievale, 2 voll., Torino, Einaudi, 2005, vol. I, rispettivamente alle pp. 317ss. e 322-23. E per venire a un pensatore ben più celebre: nell'Islam andaluso Averroè si esprime anch'egli contro il jihād: la pace è a tutto preferibile, e comunque, secondo il diritto, durante una guerra non si possono assassinare donne e bambini.

15 Cfr. F. Daftary, The Ismà îlı̄s. Their history and doctrines, Cambridge, Cambridge University Press, 1990, pp. 124; cfr. anche B. Lewis, An Epistle on Manual Crafts, in "Islamic Culture", 17, 1943, pp. 141-151; Y. Marquet, La place du travail dans la hiérarchie ismā îlienne d'après L'encyclopédie des Frères de la Pureté, in "Arabica", 8, 1961, pp. 225-237. 
Vorrei anche ricordare la tesi per cui proprio l'elitarismo è stato considerato fra le principali cause della lontananza dei filosofi dalla militanza política: ${ }^{16}$ gli studiosi ritengono che, in genere, i modelli di "città ideale" che fioriscono in ambiti sciiti - come quelli di al-Fārāâ̄ e dei "Fratelli della Purità" - siano puramente teorici, allegorie, cioè, di "città della conoscenza" che possono essere anche considerate "città di Dio" se, come ho detto, l'obiettivo finale della filosofia è la conoscenza divina. ${ }^{17}$

E che Dio sia il vero obiettivo dell'intera ricerca filosofica lo dimostra l'assetto enciclopedico di grandissima parte dei sistemi filosofici islamici, e soprattutto la raccolta delle cinquantuno epistole attribuite agli Ikhwān al-Șafă' (o "Fratelli della Purità"), una vera e propria enciclopedia sciita (se non addirittura ismailita) delle scienze. Alla base dello studio delle singole discipline sta infatti il convincimento che nei vari aspetti della natura vadano rintracciati i "segni" per comprendere Colui che l'ha creata. Tutte le scienze sono dunque considerate come gradi successivi per giungere alla verità religiosa; e "segni" in arabo si dice ayāt, con lo stesso termine che designa i versetti del Corano. Almeno fino ad Avicenna, inoltre, anche la filosofia islamica sostenne l'idea della finitezza dell'universo e, con qualche eccezione, la dottrina del geocentrismo. In tal quadro, sono importanti i versetti coranici in cui si dice che "Dio insegna ad Adamo i nomi delle creature" (Sura II, vv. 30-31): nel momento in cui si riconferma come maestro supremo, il Creatore fa con ciò nello stesso tempo di Adamo il "primo profeta", ponendolo quale depositario di una conoscenza profonda delle vere nature delle cose, nature che, com'è ben noto, ai nomi sono sottese.

Anche il sufismo considera il creato come il mezzo per arrivare a Dio, e proclama a sua volta una forma di universalismo (quando la verità è raggiunta, le differenze fra religioni perdono significato) e, insieme, di umanesimo religioso: come i filosofi avevano rivestito dell'abito del pensiero greco i concetti tradizionali della fede islamica (fede, cioè, in un Dio unico e creatore, nell'esistenza di angeli e jinn, nella profezia e nel Libro, nel particolare destino ultraterreno dell'uomo, che si concretizzerà nel giudizio e della ricompensa finale), così i sufi enfatizzano al massimo l'importanza di ḥadìth come quello che attribuisce a Dio il detto: "Ero un tesoro nascosto e volli essere rivelato". ${ }^{18}$ Ogni cosa nell'universo riflette uno dei nomi di Dio, cioè uno degli aspetti dell'Assoluto; prese nel loro insieme, le creatu-

${ }^{16}$ Cfr. J.L. Kraemer, The jihād of the falāsifa, in "Jerusalem Studies in Arabic and Islam", 10, 1987, pp. 288-324 e, più recentemente, S. Stroumsa, Philosopher-king or philosopher-courtier? Theory and reality in the faläsifa's place in Islamic society, in C. de la Puente (ed.), Identidades marginales (Estudios onomástico-biográficos de al-Andalus, XIII), Madrid, Consejo Superior de Investigaciones Cientificas, 2003, pp. 433-59.

17 Nell'occidente andaluso - ove regimi religiosamente più restrittivi impediscono lo sviluppo della filosofia, tale elitarismo sfocia nel dramma del "solitario" (mutawaḥhid) di Ibn Bājja - che rischia di optare addirittura per il suicidio -, o in modelli "politici" che, fino ad Averroè, hanno esiti soltanto negativi.

18 "Per questo", sembra sottintendere Dio, "ho creato il mondo". 
re costituiscono un intero che corrisponde alla coscienza di quell'Assoluto. Ma senza l'essere umano l'universo non può rappresentare un intero: solo l'uomo è colui che sintetizza tutte le forme dell'essere, egli è la causa dell'apparizione del mondo: "Se non fosse per te, non creerei il cielo", dice il Signore. Per il sufismo, dunque, vale il concetto del mondo come "macrantropo": il mondo vi si rivela infatti come riflesso dell'uomo.

I riferimenti al Libro sacro ci danno occasione di rimarcare che anche il culto della lingua araba (sia pur talora avvicinato a quello del latino presso gli umanisti) ha, in Islam, radici religiose: l'arabo è la lingua di Dio, e il Sacro Corano il Dio "inlibrato" (come per i cristiani si parla di un Dio "in-carnato"), l'altro modo cioè, accanto alla Natura, col quale il Creatore si fa conoscere agli uomini. E se è vero che anche dopo la disintegrazione dell'Impero musulmano i fedeli continuarono ad essere accomunati - in modo sia pur relativo - da una sola lingua letteraria comune, da una sola legge, da una sola cultura, ciò è sempre assicurato dal fatto che l'arabo è "lingua sacra", e che la storia è in genere ${ }^{19}$ una "ierostoria".

$* * *$

La nostra disamina dovrebbe, a questo punto, aver evidenziato che il carattere di base della grande cultura islamica, pur nella sua varietà, è la sua strettissima e costante interrelazione con la religione. Insomma, credo si possa ben dire che per i filosofi e gli scienziati musulmani l'essere umano è al centro di tutto, ma sempre in quanto anch'esso è "califfo di Dio sulla terra" o, per dirla in termini filosofici, un "microcosmo", cioè una riproduzione dell'intera opera divina, un compendio della creazione. E quando - ma solo nell'Ottocento - l'Islam ritenne di poter parlare esso stesso di un suo "rinascimento", concetto che espresse col ben noto termine di nahḍa, lo spirito di tale rinascimento venne ancora ravvisato in un ritorno alla religione delle origini, in un riallacciarsi alla purezza iniziale a fronte delle corruttele allora esistenti. Proprio questo, si riteneva, avrebbe riportato l'Islam alla primitiva grandezza; e sulla stessa linea Muḥammad 'Abduh enfatizzò il ruolo giocato, per il progresso europeo, dal Protestantesimo, con la sua reazione ad un Cattolicesimo decaduto e corrotto. ${ }^{20}$

Ora, se i modelli islamici, anche quelli filosofici, sono eminentemente religiosi (e sebbene, naturalmente, ciò non privi l'uomo della sua grandezza come privilegiato destinatario del messaggio - del sapere - divino, né i vari sistemi filosofici della loro rilevanza teoretica), parecchi dei concetti contrabbandati ultimamente dai "nuovi umanisti" per fondare un dialogo, un'apertura all'Islam, non appaiono del tutto condivisibili né dal punto di vista storico né da quello ideologico e/o dottrinale.

Con la parziale eccezione di Ibn Khaldūn, che vive tuttavia nella Spagna del XIV secolo.

Cfr. Muhammad 'Abduh, Trattato sull'unicità divina. Risalat at-Tawhid, a cura di G. Soravia, Bologna, il Ponte, 2003, p. 165. 
Soprattutto, non sembra che possa essere l'etica il piano sul quale giocare il dibattito fra Islam e Occidente, che grande parte dell'intellighenzia islamica - non solo i fondamentalisti - considera oggi come il nemico assoluto. Si tratta di intellettuali di varia provenienza, che ritengono che il XXI secolo vedrà la fine della supremazia occidentale e, conseguentemente, la rinascita dell'Islam; secondo loro, nel vuoto lasciato in Occidente da Ebraismo e Cristianesimo l'Islam può entrare e vincere la partita, non subendo la modernità, bensì islamizzandola; vogliono far emergere valori di matrice islamica ma con vocazione universale, che prenderanno il posto di quelli, ormai decaduti, dell'Occidente. Per questi intellettuali, che talora non disdegnano neppure l'ideale del dialogo interreligioso, il vero umanesimo ha la sua base nella rivelazione coranica, e sia direttamente, sia attraverso la mediazione di tale umanesimo, la "città islamica" potrà instaurarsi sulla terra. Tali intellettuali insistono d'altro canto anche sulla ricchezza, troppo a lungo ignorata, dei grandi pensatori del loro passato, così come ravvisano nel razionalismo laico di matrice illuministica una delle principali cause dell'attuale decadenza dell'Occidente.

Gli studiosi occidentali, da parte loro, rimproverano all'intellighenzia musulmana di non collocare i grandi pensatori islamici nel loro inscindibile rapporto col pensiero, non solo greco, ma anche ebraico e cristiano - il che solo consentirebbe di ravvisare in essi, ad onta della netta impronta teologizzante che ho sopra illustrato, una delle fonti dell'umanesimo del '400. Peraltro, si deve con amarezza osservare che sebbene la consapevolezza dell'immenso debito che noi abbiamo verso il mondo islamico per il recupero e la trasmissione dei testi classici dovrebbe essere ormai un dato acquisito, probabilmente non è più così per le più giovani generazioni, bombardate dai media e dai pronunciamenti di quegli intellettuali la cui importanza è spesso vergognosamente enfatizzata, e che nella foga polemica arrivano a negare persino l'evidenza. Ma anche dove la grandezza filosofica e scientifica del passato siano riconosciute all'Islam, l'Occidente laico tende a negargli importanza dal punto di vista religioso e morale.

Una posizione così ambigua non ci aiuta certo a capire l'Islam, e riperpetua, ancora una volta, gli antichi stereotipi che ebbero origine nel Medioevo, ma ai quali certo non rinunciò l'umanesimo del '400. Naturalmente, non si può sottacere che la "città islamica" auspicata da taluni esponenti di quell'intellighenzia è in realtà una teocrazia, si rifà cioè a un modello ormai irrimediabilmente estraneo all'Occidente, anche ove volesse essere instaurato da parte cristiana.

In realtà, bisognerebbe prendere atto del fatto che il razionalismo greco che ha integrato le matrici semitiche del Cristianesimo non è quello degli umanisti, come non lo è il "razionalismo" dell'Islam del periodo aureo, mentre è proprio con questo razionalismo che molti intellettuali musulmani fanno oggi i conti, alla ricerca di una sua nuova interpretazione che li ponga in grado di affrontare le sfide della modernità, nei confronti della quale essi lasciano spesso intravedere - ad onta delle indicazioni e delle fatwe dei "ulema - un approccio di tipo "selettivo". 
Mi sento dunque di condividere la linea di quegli studiosi musulmani che ritengono che un ripensamento del passato islamico, alla ricerca di una sua nuova interpretazione confacente alla modernità, non possa prescindere da una nuova ermeneutica del Libro sacro. Tale ermeneutica, naturalmente, non è certo destinata a rispondere all'interrogativo dell'Occidente, se l'Islam si presterà ad evolversi in senso razionalista e modernista. Una simile ricerca mira piuttosto, da caso a caso, a ripensare storicamente l'origine dell'Islam e del messaggio coranico, a riconsiderarne i contenuti alla luce delle nuove scienze antropologiche e sociali, ad enuclearne un messaggio universale al di là delle impostazioni strettamente confessionali. ${ }^{21}$

Mi auguro di essere riuscita a indicare alcune linee guida, storicamente e ideologicamente fondate, per ricostituire un dialogo con l'Islam di oggi nel rispetto delle sue istanze specificamente religiose e spirituali. La conclusione più feconda che a mio parere i "nuovi umanisti" potrebbero recepire, sulla base di una valutazione informata e intellettualmente onesta della storia e della civiltà islamica, e che può essere condivisa dall'Occidente, laico e non, è che soltanto l'Islam ha il diritto, dal suo interno, di formulare (o eventualmente riformulare) un ideale umanistico conciliabile con i propri valori religiosi, che consenta al contempo di fruire di alcuni aspetti del "progresso" della modernità.

Il "nuovo umanesimo", peraltro, non sottovaluta affatto il rapporto con la religiosità e la trascendenza, e le comunità musulmane si sono spesso poste esse stesse quali possibili interlocutrici in tale processo di integrazione. Una migliore conoscenza reciproca, attraverso gli universali valori dell'intelligenza e della cultura, comporterà di certo un mutuo arricchimento dei rappresentanti delle diverse religioni. $\mathrm{Ma}$, in nome della grandezza dell'uomo, riusciremo forse insieme pure a scoprire un modo per comporre la varietà delle diverse "ragioni". Nel Corano, del resto (Sura XXXVIII, v. 73 ss.), gli angeli, a parte Iblis, ${ }^{22}$ adorano l'uomo. E ciò può essere interpretato - anche - come un riconoscimento dell'alto valore della ragione umana.

\footnotetext{
${ }^{21}$ Al di là delle pionieristiche posizioni di Mohammed Arkoun, si veda la recente disamina di $\mathrm{R}$. Benzine, Les nouveaux penseurs de l'islam, Paris, Michel, 2004. Per certi versi, le posizioni di molti pensatori islamici di oggi potrebbero essere accostate alle preoccupazioni, espresse dal Pontefice cattolico, sui rischi connessi al culto assoluto della tecnica, senza aperture alla spiritualità.

22 Per questo, secondo alcuni mistici, Lucifero è il supremo teorizzatore dell'assoluto monoteismo.
} 